\title{
LAS TIC, EL PROCESO DEL CONOCIMIENTO Y LAS COMPETENCIAS DOCENTES
}

\author{
Augusto Perez Lindo*
}

Recebido: 9 jun. 2014

Aprovado: 31 jul. 2014

*Universidad Nacional del Este. Paraguay. E-mail: perezlindo@gmail.com

Resumen: En el marco del Seminario Internacional "Formación y conocimiento" organizado por la Universidad de Sorocaba presentamos un análisis de los efectos de las TICs en los procesos del conocimiento. Tratamos de demostrar que esos efectos se producen en varias direcciones: cambios en la visión de la realidad, de la subjetividad, del lenguaje, de los paradigmas, de las relaciones sociales. Propone fortalecer competencias adecuadas al cambio de paradigma, como la capacidad para seleccionar e interpretar informaciones, la capacidad para pensar científicamente, la capacidad para comunicar, la capacidad para convivir, la capacidad para dominar varios lenguajes.

Palabras claves: TICs. Conocimiento. Competencias.

\section{THE ICTS , THE PROCESS OF KNOWLEDGE AND THE TEACHING SKILLS}

Abstract: In the framework of the International Seminar "Teacher Training and Knowledge" (oct.2014) organized by the University of Sorocaba we present an analysis of the impact of ICTS in the processes of knowledge. We try to demonstrate that these effects are produced in several directions: changes in the perception of reality, of subjectivity, in the use of language, on the paradigms and on social relations. We propose to improve suitable skills for the change of paradigm, i.e. the ability to search and interpret information; the ability to think scientifically, the ability to communicate, the ability to live together, the ability to learn languages.

Key words: ICTS. Knowledge; Skills.

\section{AS TICS, O PROCESSO DE CONHECIMENTO E AS COMPETÊNCIAS DOCENTES}

Resumo: No marco do Seminário Internacional "Formação e Conhecimento" organizado pela Universidade de Sorocaba apresentamos una análise dos efeitos das TICs nos processos do conhecimento. Tratamos de demonstrar que esses efeitos se produzem em várias direções: mudanças na visão da realidade, da subjetividade, da linguagem, dos paradigmas, das relações sociais. Propõe fortalecer competências adequadas à mudança de paradigma, como a capacidade para selecionar e interpretar informações, a capacidade para pensar cientificamente, a capacidade para comunicar, a capacidade para conviver, a capacidade para dominar várias linguagens.

Palavras-chave: TICs. Conhecimento. Competências. 


\section{EL NUEVO ORDEN DEL CONOCIMIENTO}

Podría afirmarse que los cambios que están en curso afectan a todas las disciplinas en cuanto que se modifica la idea de la realidad y de la verdad. Las ideas sobre la materia, sobre el ser viviente, sobre las identidades humanas o sobre los procesos económicos se han vuelto menos concretas, se han "desustancializado" o "desmaterializado" como algunos prefieren decir (PEREZ LINDO, 2003).

Las tecnologías de la información y comunicación (las TIC como se las denomina) vienen produciendo muchos efectos que han sido comentados o estudiados de diversas maneras. Vamos a analizar en particular los efectos de las TIC en los procesos del conocimiento y las consecuencias para la educación en general, y en particular para la enseñanza universitaria.

\section{¿LAS TIC MODIFICAN LOS PROCESOS DE CONOCIMIENTO?}

Cuando Marshall McLuhan publicó "Understanding Media" (1996) planteó por primera vez la nueva situación de la inteligencia humana frente a los impactos de la televisión, la radio y el cine, o sea, la cultura audiovisual. Y observó que las tecnologías tienden a manifestarse como extensiones del cuerpo humano. Los medios de comunicación de masas tienden a reemplazar la memoria y la inteligencia colectiva.

Con Internet y con los sistemas de información que circulan en distintos medios (computadoras, celulares, IPADs, tabletas) se avanza más profundamente. Esto provoca el interrogante y el libro de Nicholas Carr ¿Qué está haciendo Internet con nuestras mentes? (CARR, 2011). Desde el comienzo describe su dramática situación:

Los beneficios son reales. Pero tienen un precio. Como sugería McLuhan los medios no son sólo canales de información. Proporcionan la materia del pensamiento, pero también modelan el proceso de pensamiento. Y lo que parece estar haciendo la Web es debilitar mi capacidad de concentración y contemplación (p. 18).

El creador del concepto de "realidad virtual", Jaron Lanier, reflexiona alarmado sobre las tendencias a colocar las redes y los sistemas de información por encima de la creatividad de los individuos. En su libro "No somos computadoras" (LANIER, 2012) lo que se propone rescatar la idea de persona que implica nuestra inteligencia operando sobre las computadoras. 
Queremos sostener aquí que las TIC provocan tales cambios en los procesos cognitivos que estamos obligados a revisar las teorías del conocimiento que circulan en el mundo académico y escolar. De manera general todos los individuos actuales deberían asumir que las tecnologías de la comunicación y de la información han modificado nuestras relaciones con la sociedad, con la naturaleza y con nosotros mismos. Los procesos cognitivos no son más que indicios de este fenómeno.

Desde una perspectiva compleja podemos entender el proceso del conocimiento como una interacción permanente entre las siguientes dimensiones: la realidad, el sujeto, las ideas, el lenguaje, la sociedad (PEREZ LINDO, 2010).

Aunque la epistemología contemporánea todavía siga dominada por la tendencia a encontrar un principio unívoco, nosotros sostenemos que en los procesos cognitivos intervienen con eficacia equivalente la realidad, la subjetividad, las ideas, el lenguaje y el consenso social (PEREZ LINDO, 2010).

Asumimos, pues, como un punto de partida, que todo conocimiento puede formarse o legitimarse desde cualquiera de las dimensiones arriba descriptas. Esta perspectiva multidimensional e interactiva converge con la teoría de las inteligencias múltiples de Howard Gardner (2003). Trasciende las visiones monistas o dualistas. Se aproxima a la visión compleja del proceso del conocimiento que sostiene Edgar Morin (1986).

\section{¿SE PUEDE SEGUIR SIENDO REALISTA EN LA ERA DE INTERNET?}

Durante los milenios que forman parte de la historización humana siempre subyacía la creencia de que para conocer debíamos ser capaces de representarnos la naturaleza y sus estados. O sea, el realismo representacionista.

Actualmente, la visión científica sobre sobre el estado del universo depende de los datos que se acumulan en los sistemas de información. Un astrónomo no necesita mirar el espacio desde el telescopio: encontrará más información en las bases de datos de sus computadoras. El estado del tiempo, de los aeropuertos, del sistema financiero, de los mares o de las cosechas los conocemos a través de los sistemas de datos, de satélites o de registros electrónicos. La naturaleza ha quedado mediatizada por los sistemas de información.

Ahora los sistemas de información están penetrando el cuerpo humano llegando a los determinantes genéticos de cada individuo con la capacidad 
de anticipar enfermedades futuras (RIFKIN,1999). La "realidad" del cuerpo humano ya no depende de la mirada del médico ni tampoco de sus aparatos tradicionales. Las biotecnologías van más allá de lo visible. Los sistemas de información disponen de una visión del mundo subyacente a la que pocos expertos pueden llegar.

Por otro lado, está lo que se llama comúnmente la "realidad virtual". En este caso lo "virtual" se suele oponer a lo "presencial". Dos personas pueden tener interacciones "virtuales" mediante computadoras, pantallas, robots y otros dispositivos. Un médico puede dirigir "virtualmente" una operación "a distancia" de un paciente situado muy lejos de sí. Existen consultorios psicoterapéuticos a distancia.

"A distancia" no es lo mismo que "virtual". De hecho, la enseñanza "virtual" tiende a superar a la "educación a distancia" que algunos consideran "tradicional". ¿Por qué? Porque la "virtualidad" permite una interacción tanto o más intensa que la "presencialidad".

Los sistemas de información representan a la realidad en formatos virtuales que permiten observar los fenómenos o procesos (desde el universo hasta los genes, desde el cuerpo humano a las superficies urbanas) de una manera más profunda.

Se habla de "realidad virtual" pero de hecho estas concepciones de la realidad se podrían considerar como "hiper-realistas". El "conocimiento duro" de los sistemas de información, o sea, el constituído por los datos objetivos de las cosas, constituye una dimensión esencial del mundo en que vivimos. Cuando perdemos la conectividad con el "info-mundo" muchas actividades se paralizan.

El conjunto del sistema de información constituye una nueva dimensión: realidad virtual, sistema de datos, matrix, ciberespacio, cibercultura. Una nueva dimensión que se incorpora al conjunto de objetos que forman parte de la "realidad analizable", aunque no sea, "observable". La naturaleza y la sociedad la percibimos a través del prisma de esta nueva tecno-estructura.

Este conflicto, entre la vigencia de una idea de la realidad anterior frente al surgimiento de una "nueva realidad" provoca disociaciones que tienen efectos en la política, en la economía y en el comportamiento de los jóvenes. Los sistemas educativos todavía viven en el mundo pre-informático pero de hecho se encuentran alterados por la cultura de las TIC. La incongruencia entre la cultura escolar tradicional y la nueva realidad es una de las causas del malestar en las instituciones educativas. 


\section{EFECTOS DE INTERNET SOBRE LOS SUJETOS Y LAS SUBJETIVIDADES}

Las TIC producen efectos neuronales y alteran el funcionamiento de los sentidos. En muchos casos se están empleando para suplir las deficiencias físicas o psicológicas de las personas de manera sorprendente. Ciegos que ven, sordos que escuchan, personas paralizadas que realizan actividades, corazones que palpitan por injertos electrónicos, son algunos de los milagros de la electrónica y la informática.

Se ha señalado en otro sentido que la cultura audio-visual desplaza a la reflexividad a favor de las imágenes o de los estímulos audiovisuales. Resulta interesante redescubrir en el libro clásico de André Leroi Gouhran (1964) "El gesto y la palabra" que en el proceso evolutivo comenzamos a hablar con las manos y que el desarrollo de los sentidos nos fue permitiendo crear una cultura simbólica en la que surge la civilización. El actual predominio de las TIC recrea el uso primitivo de las manos, privilegia la visión y el sonido sobre la reflexión, modifica la manera de crear o recrear el mundo simbólico de los humanos.

Desde la perspectiva de la Inteligencia Artificial autores como Marvin Minsky (1987) afirman que el proceso evolutivo puede conducir a la superación de la mente humana por los sistemas de información. De hecho, ya existen muchos simuladores del cerebro humano y los modelos de inteligencia artificial se aplican en múltiples menesteres. Las TIC hacen posible el surgimiento de un mundo "post humano", algo que ya se escenifica en la literatura y el cine "ciberpunk".

Antes de Internet ya existía una tensión entre la cultura audio-visual y la cultura del "pensamiento escrito" (para llamarla así). Se publicaron ensayos y críticas sobre la "inteligencia visual", sobre la "masificación" del pensamiento a través del cine, la radio y la televisión.

A nivel de la memoria Internet permite crear una "memoria exogámica" sobre la cual comienza a reposar el desempeño intelectual, laboral, artístico o económico de los individuos. Lo que antes era necesario memorizar ahora se encuentra disponible en los archivos electrónicos.

A nivel de las relaciones intersubjetivas Internet abre camino al Chat, al correo electrónico, facebook, twitter y a numerosas redes sociales electrónicas capaces de recrear la socialidad de una manera especial. Surge una socialidad virtual que permite expresar sentimientos de amistad, de odio, de pasión, de intereses intelectuales compartidos, etc. La subjetividad y la intersubjetividad 
virtual no solo modifican las relaciones humanas sino también a los sujetos mismos.

Casi todos los niños actuales están rodeados de amigos virtuales. Se forman "tribus" virtuales que se organizan para escenificar guerras o para apoyar un grupo de rock, o un equipo de football o un movimiento político. Internet permite compartir estilos de vida entre jóvenes y adultos de cualquier lugar del mundo. Las redes están reemplazando los anteriores agentes de socialización: la familia, el Estado, las iglesias, los partidos políticos, los sindicatos, los clubes.

La interacción con Internet crea discontinuidades en el pensamiento de los sujetos. Cambia el modo de asimilación de informaciones y de conocimientos. El sistema de información condiciona el funcionamiento de la inteligencia y los usuarios informáticos ya sufren diversas perturbaciones (CARR, 2011; GARDNER, 2014).

\section{EFECTOS DE INTERNET EN LOS SISTEMAS DE IDEAS (NOOSFERA)}

Los sistemas de información crean una nueva Noosfera que no solo abarca todas las bibliotecas del mundo, sino todas las publicaciones y los registros de información desde los más primitivos hasta los más avanzados del espacio cósmico.

Una de las pioneras iniciativas para sistematizar la información científica mundial fue la de Eugene Gartfield que creó el Institut for Scientific Information (ISI) en 1960. Fue a nuestro entender el verdadero precursor de Google. Logró repertoriar millones de artículos de revistas científicas en todo el mundo, en todos los idiomas. Creó boletines periódicos, como los "Currents Contents" de las diversas disciplinas y permitió por primera vez en la historia humana tener un mapa global de la producción científica de la Humanidad.

En la actualidad tenemos Google y otros buscadores que en forma casi instantánea nos brindan una lista interminable de todas las referencias que existen respecto a cualquier tema que nos interesa.

Normalmente cuando consultamos por un tema nos aparecen miles de referencias que nos dejan abrumados. Aquí nos encontramos con un efecto que ya había previsto Gartfield: la explosión de informaciones y la necesidad de encontrar algunos criterios para organizarla, seleccionarla e interpretarla.

Otro evento de fundamental fue la creación de la Wikipedia, o enciclopedia libre, que comenzó en 2001. La Wikipedia actual constituye la más grande fuente de conocimientos que exista en la Humanidad. Aunque muchos duda- 
ban de su valor como referente académico su desarrollo llevó a desplazar y eliminar las enciclopedias impresas en papel como la famosa Enciclopedia Británica.

Las TIC no solo han alterado las compartimentaciones de las distintas culturas, no solo han reemplazado paulatinamente a miles de bibliotecas, no solo han permitido universalizar el acceso a todos los conocimientos sino que además interfieren activamente en la formación de nuevos imaginarios a través de sus productos y de las interacciones de los usuarios. Los usos del chat, de Facebook y del Twitter reflejan esta tendencia. Ha surgido un nuevo imaginario global que de manera a veces difusa y confusa moviliza a los individuos y a las masas.

\section{EFECTOS DE INTERNET EN LOS USOS DEL LENGUAJE}

\section{Raffaele Simone observa lo siguiente:}

a finales del siglo XX hemos pasado gradualmente de un estado en el que el conocimiento evolucionado se adquiría sobre todo a través del libro y la escritura (es decir, a través del ojo y la visión alfabética $\mathrm{o}$, si se prefiere, a través de la inteligencia secuencial) a un estado en el que éste se adquiere también - y para algunos principalmente - a través de la escucha (es decir, el oído) o la visión no-alfabética (que es una modalidad específica del ojo), es decir, a través de la inteligencia simultánea (2000, p. 37).

Simone no es el primero que destaca el desplazamiento de la lectura por los estímulos audiovisuales. Ya lo había hecho McLuhan. Para comprender las consecuencias de este hecho hay que vincularlo con el cambio en el modo de procesar los conocimientos.

En el Ciclo Básico Común de la Universidad de Buenos Aires se constató que en promedio el capital lingüístico de los ingresantes giraba en torno a 300 palabras. Constataciones semejantes se han hecho en Estados Unidos, en Brasil y en otros países. Al punto que en Alemania una norma estableció hacia el 2000 que todos los egresados de la escuela secundaria debían dominar por lo menos 1.500 palabras.

Estas manifestaciones de pérdida del capital lingüístico en el mundo occidental se relacionan con la frecuentación intensiva de la televisión y ahora de la computadora. Se ha estimado que un niño a los 12 años pasó más de 12.000 frente al televisor y la computadora, mientras que en muchos países latinoamericanos apenas ha pasado 10.000 horas con sus maestros. 
Analizando algunos informes y artículos especializados se puede constatar que la omnipresencia de Internet, de la televisión y del celular entre los jóvenes tiene consecuencias evidentes, verificables, en el abandono de la lecto - escritura, en la pérdida del capital lingüístico y en la conformación de un nuevo tipo de discurso fundamentalmente narrativo.

\section{CONOCIMIENTO Y SOCIEDAD EN LA ERA DE INTERNET}

En la teoría de Jûrgen Habermas sobre la "acción comunicativa" se supone que la formación de conocimientos ciertos depende de un consenso intersubjetivo que se apoya en reglas argumentativas. Se ha dicho que esta tesis resulta idealista y de hecho podemos considerarla como una idealización de los acuerdos intersubjetivos que producen las comunidades científicas.

¿En qué sentido Internet y las redes de información fortalecen o debilitan la cultura científica en la sociedad? ¿En qué sentido se fortalecen o debilitan las capacidades sociales para producir y legitimar los conocimientos científicos? Para responder a estas debemos tener en cuenta algunas experiencias y evidencias significativas:

- con las bases de datos, los sistemas de información y las enciclopedias electrónicas todo el mundo tiene un acceso infinito a los estudios de cualquier disciplina;

- Los programas informáticos permiten controlar en parte la explosión de conocimientos que se da a escala mundial mediante la creación de selectores e intérpretes de todo tipo;

- Internet y los programas inteligentes han multiplicado miles de veces la capacidad de observación científica en todas las disciplinas; hoy existe sobreabundancia de información y escasez de teorías y métodos para aprovecharlas;

- en las ciencias biológicas, en astrofísica, en geología y otras disciplinas el desarrollo de programas e instrumentos informáticos ha permitido acortar los tiempos de investigación y ha incrementado la capacidad para procesar los datos;

- como toda la información científica circula ahora por Internet a través de las revistas científicas se ha multiplicado varias veces la capacidad de control, evaluación, contrastación, de las informaciones y de las teorías; 
- por la misma razón, se ha terminado con la compartimentación de las ciencias y de los países en materia científica acelerando los intercambios y permitiendo a los países más atrasados cortar la brecha de conocimientos con los países más avanzados.

Desde un punto de vista epistemológico la experiencia actual amplía la noción de "comunidad científica" antes centrada en los países más desarrollados que monopolizaban las principales fuentes de información.

Los movimientos sociales (como los ecologistas, los defensores de las culturas regionales o ancestrales, los defensores de los derechos humanos y muchos otros) han encontrado en Internet y en las redes una plataforma adecuada para defender sus derechos o para confrontar a grupos o intereses dominantes. En este sentido la democratización del conocimiento que hace posible Internet también le dio a la cultura científica una nueva oportunidad para actuar como un agente emancipador.

\section{REFORMA EPISTEMOLÓGICA Y PEDAGÓGICA PARA SITUARSE EN LA ERA DE LAS TIC}

¿Cómo aprovechar todo el potencial que ofrecen las TICs para mejorar la enseñanza y los aprendizajes? ¿Cómo evitar los daños colaterales que produce la cultura masiva de las TICs? ¿Qué competencias deberían desarrollar tanto los profesores como los alumnos?

Si lo que venimos diciendo es verosímil deberíamos por lo menos revisar nuestras ideas sobre la realidad y nuestras teorías del conocimiento. Millones de personas manejan sus relaciones con el espacio y la geografía a través del GPS. Del mismo modo los profesores deberían asumir que las nociones del espacio y del tiempo, de los objetos, de los cuerpos, de las relaciones sociales, se vienen modificando en las últimas décadas. Deberíamos compartir la ontología hiper-realista que adoptan los nativos digitales: todo lo virtual es real, todo lo real es virtual.

Debemos asumir que disponemos de una teoría más compleja del conocimiento que en el pasado gracias a las críticas epistemológicas, a las neurociencias, a la psicología cognitiva, a la sociología del conocimiento. Nuestras universidades, todavía, enseñan a pensar de manera monodisciplinaria: los médicos, los contadores, los arquitectos, los ingenieros, los abogados, etc. se forman con profesionales que aprendieron los mismos esquemas reduccionistas, la misma división del trabajo intelectual, que inventó la universidad 
tradicional europea. La reforma de la educación superior europea luego de los Acuerdos de Bolonia (1999 - 2010) permitió introducir la formación transdisciplinaria entre los 54 países que adhirieron al programa.

Los nuevos paradigmas y los nuevos contextos reclaman nuevas competencias para los docentes (PEREZ LINDO, 2012). Esto quiere decir que tenemos que tomar en cuenta los impactos producidos por las TICs y por otros factores. Desde el ángulo de nuestro objeto (las TIC) resulta evidente que todos los profesores, en todos los niveles, necesitan una cultura informacional. Que implica no solamente la alfabetización informática sino también la capacidad para buscar, seleccionar e interpretar información con las fuentes que ofrecen las Redes de Internet.

Cabe prevenirse contra la ilusión de que el acceso a las computadoras o a las redes de información incrementa por si mismo la capacidad de aprendizaje. Necesitamos conocimientos específicos para acceder a la información y para aprovecharla en los aprendizajes. Esto lo demostró claramente la experiencia del Plan Ceibal en Uruguay y es evidente en las experiencias de Argentina o Brasil tendientes a dotar con computadoras a todos los alumnos.

Todo esto nos indica que desde el punto de vista de la formación docente tenemos que disponer de por lo menos una decena de competencias básicas: capacidades científicas, lingüísticas, informacionales, comunicativas, metodológicas, pragmáticas, pedagógicas (PEREZ LINDO, 2012). En cualquier nivel y en cualquier contexto.

Docentes con estas competencias logran desde lugares marginales o pobres que sus alumnos aprendan adecuadamente y que accedan de una u otra manera a los recursos informáticos por Internet o por telefonía o por televisión. Existen redes de educación a distancia por radio-televisión, por Internet o por Facebook. Pero la competencia pedagógica sigue siendo esencial.

Desde un punto de vista filosófico el Sistema de Información favorece la pluralidad de pensamientos y también la contrastación de teorías divergentes. Por otro lado, Internet aproxima todas las culturas y hace posible pensar el mundo de manera global. En este sentido, Internet permitiría quebrar el etnocentrismo aún dominante en el mundo universitario global (ALTBACH, 2014).

Por otro lado, varios autores señalan que la frecuentación de Internet no favorece la reflexión ni el pensamiento argumentativo. Puede alterar la creatividad personal en la medida en que favorece la copia de otras ideas y experiencias. También deja abierto el camino para defender teorías irracionales, conjeturas inverificables y valores contrarios a la dignidad humana. 
Existen diversos caminos didácticos para utilizar las TIC. En algunos casos predominan los procedimientos técnicos, en otros la comunicación virtual y en otros el acceso a la información. La pedagogía informática se está construyendo y los estudios sobre los efectos de las TIC nos permitirán mejorar su aprovechamiento en la educación. Por ahora, no hay un modelo standard, no hay recetas universales. Todos estamos aprendiendo a transitar los mil caminos que nos abren las tecnologías de la información y de la comunicación. Lo mejor que podemos esperar de las TIC es que nos enseñe a todos a ser buenos aprendices.

\section{REFERENCIAS}

ALTBACH, Philip G. "OOCs as neocolonialism: who controls knowledge? International Higher Education, Boston, n. 75, p. 5-7, 2014.

CARR, N. ¿Qué está haciendo Internet con nuestras mentes? Superficiales. Buenos Aires: Taurus, 2011.

GARDNER, H. La inteligencia reformulada. Las inteligencias múltiples en el siglo XXI. Barcelona : Paidós, 2003.

GARDNER, H.; DAVIS, K. La generación APP. Cómo los jóvenes gestionan su identidad, su privacidad y su imaginación en el mundo digital. Buenos Aires: Paidós, 2014.

GOUHRAN, A. L. Le geste et la parole. I. Techiniques et langage. Paris : Albin Michel, 1964.

LANIER, J. No somos computadoras. Un manifiesto. Buenos Aires: Debate, 2012.

McLUHAN, M. Understanding media. The extensions of man. New York: McGraw-Hill, 1964. Traducción castellana: Comprender los medios. Las extensiones del ser humano. Buenos Aires: Paidós, 1996.

MINSKY, M. La sociedad de la mente: la inteligencia humana a la luz de la inteligencia artificial. Buenos Aires: Galápagos, 1987.

MORIN, E. La méthode. 3. La Connaissance de la Connaissance/1. Paris: Seuil, 1986. 
PEREZ LINDO, A. El concepto de realidade. Teorías y mutaciones. Buenos Aires: Proyecto Editorial, 2003.

PEREZ LINDO, A. ¿Para qué educamos hoy? Filosofía de la educación para un nuevo mundo. Buenos Aires: Biblos, 2010. Cap. 3, p. 159-175.

PEREZ LINDO, A. Competencias docentes para el siglo XXI. Buenos Aires: Tinta Fresca, 2012.

RIFKIN, J. El siglo de la biotecnología. Barcelona: Crítica, 1999.

SIMONE, R. La tercera fase. Formas de saber que estamos perdiendo. Madrid: Taurus, 2000. 